\title{
LA FAUNA DEL YACIMIENTO TUROLIENSE DE TARIEGO DE CERRATO (PALENCIA, CASTILLA-LEÓN)
}

\author{
Benigno PÉREZ, Lara AMEZUA, \\ Isabel de MIGUEL y Jorge MORALES \\ Departamento de Paleobiología. Museo Nacional de Ciencias Naturales, \\ C.S.I.C. C/. José Gutiérrez. Abascal, 2. 28006 Madrid.
}

Pérez, B., Amezua, L., Miguel, I. de y Morales, J. 2001. La fauna del yacimiento turoliense de Tariego de Cerrato (Palencia, Castilla-León). [The Turolian fauna from Tariego de Cerrato (Palencia, Castilla-León).] Revisıa Española de Paleontología, no extraordinario, 133-135. ISSN 0213-6937.

\begin{abstract}
This paper shows the fossil fauna recovered during 1995 and 1997 field works on the Tariego de Cerrato (Palencia, Duero Basin) fossil site. This site is placed in T.S.U. 4 of Mediavilla y Dabrio (1988). That fauna allowed to date with precision these sediments as Upper Turolian age.

The fossil taphonomical data indicate that the remains concentracion is due to hydrodynamic transport.
\end{abstract}

Keywords: Upper Turolian, Tariego de Cerrato, Palencia, Castilla-León.

\section{RESUMEN}

En el presente trabajo se presenta la fauna fósil recuperada en las campañas realizadas en los años 1995 y 1997 en el yacimiento de Tariego de Cerrato (Palencia, Cuenca del Duero). Este yacimiento se encuentra dentro de la U.T.S. 4 de Mediavilla y Dabrio (1988). Dicha fauna permitió datar con precisión los niveles en los que se encuentra como Turoliense superior.

De las características tafonómicas de los restos se desprende que su concentración es debida a un transporte de origen hidrodinámico.

Palabras clave: Turoliense superior, Tariego de Cerrato, Palencia, Castilla-León.

\section{INTRODUCCIÓN}

El yacimiento paleontológico de Tariego de Cerrato fue descubierto como consecuencia de los trabajos de investigación realizados por el Instituto Tecnológico Geominero de España en la Cuenca del Duero.

Desde un primer momento se apreció la importancia de este yacimiento, tanto por la asociación faunística que presenta, como por los valiosos datos biocronológicos que aporta para la Cuenca del Duero, ya que en ella son extremadamente escasos los yacimientos continentales neógenos.

Gracias a las subvenciones concedidas por la Junta de Comunidades de Castilla y León se pudieron llevar a cabo distintas excavaciones realizadas en los años 1995 y 1997.

\section{SITUACIÓN GEOGRÁFICA Y GEOLÓGICA}

El yacimiento de Tariego de Cerrato se localiza en las proximidades de la población del mismo nombre, junto a la carretera que une dicha localidad con Cevico de la Torre (Fig. 1).

Geológicamente se encuentra situado en el sector central de la Cuenca del Duero (provincia de Palencia). Mediavilla y Dabrio (1988) diferencian en este sector cuatro unidades sedimentarias (UTS) constituidas por materiales siliciclásticos, carbonatados y evaporíticos correspondientes a ambientes fluviales, palustres y lacustres cuya edad corresponde al Neógeno superior.

El yacimiento se sitúa en la UTS 4 (Mediavilla et al., 1995). La importancia de esta unidad radica en que se 


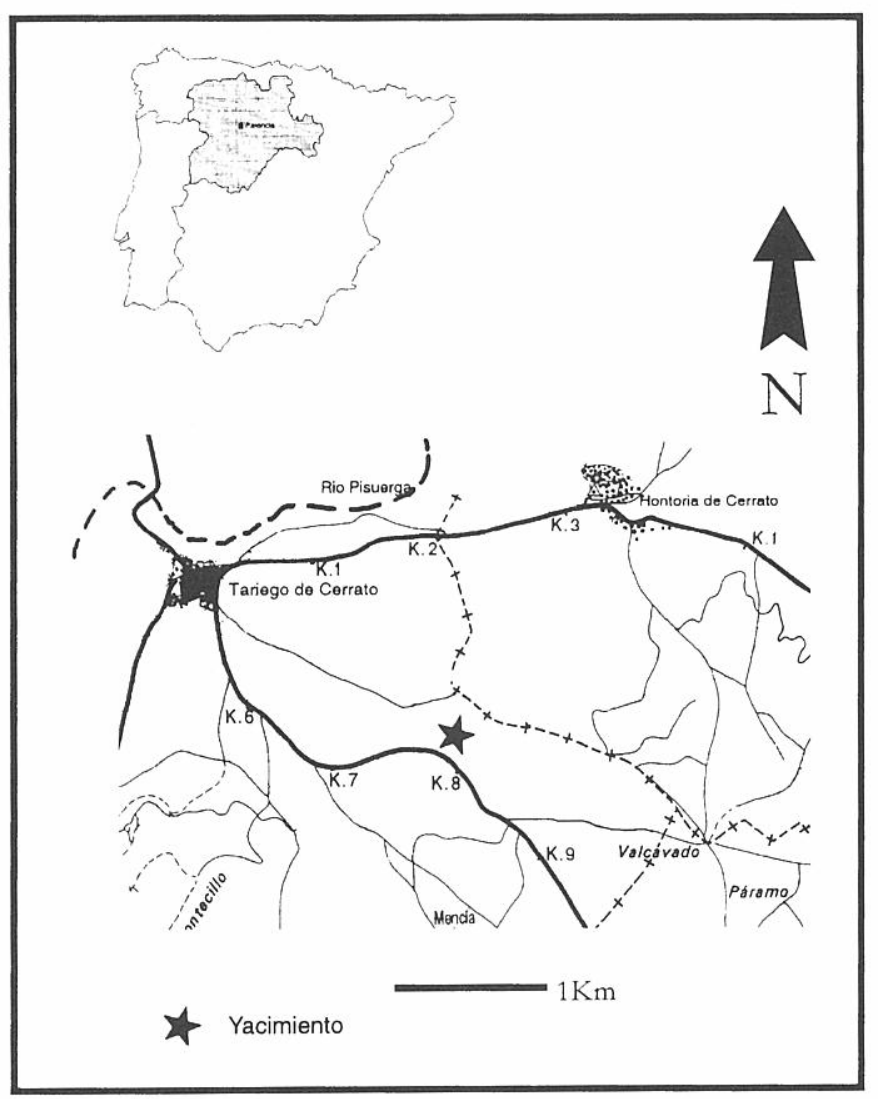

Figura 1. Situación geográfica del yacimiento de Tariego de Cerrato.

Geographical situation of the Tariego de Cerrato site.

encuentra encajada en la unidad previa y registra el inicio de la definición de la red fluvial de este sector (Fig. 2). Las diferentes edades que hasta ahora se le venían asignando a la unidad, estaban comprendidas dentro de un amplio rango que abarcaba desde el VallesienseTuroliense hasta el Plioceno.

La litoestratigrafía del yacimiento está constituida por conglomerados y arenas que pasan tanto lateral como verticalmente a arenas masivas, lutitas con intercalaciones de nódulos carbonatados y, finalmente, a calizas con restos de gasterópodos. Se interpreta como depósitos de canales fluviales y de llanura de inundación.

\section{PALEONTOLOGÍA}

El material recuperado ha proporcionado la siguiente lista faunística:

Orden Artiodactyla Owen, 1848

Familia Suidae Gray, 1821

Género Propotamochoerus Pilgrim, 1926

Propotamochoerus provincialis (Gervais, 1859)

Familia Bovidae Gray, 1821

Género Tragoportax Pilgrim, 1837

Tragoportax sp.

Bovidae indet.
Unidades

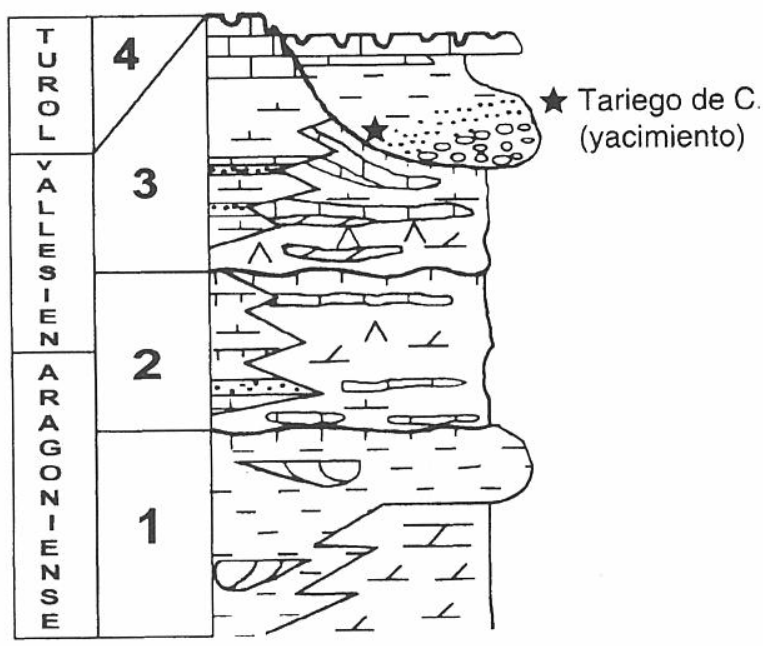

Figura 2. Columna estratigráfica para el Neógeno del Sector Central de la Cuenca del Duero (modificado de Mediavilla y Dabrio, 1988).

Stratigraphical section for central sector of Duero Basin (adapted from Mediavilla and Dabrio, 1988).

Orden Perissodactyla Owen, 1848

Familia Equidae Gray, 1821

Género Hipparion Christol, 1832

Hipparion sp.

Orden Proboscidea Illiger, 1811

Familia Gomphotheriidae Hay, 1922

GéneroTetralophodon Falconer y Cautley, 1857

Tetralophodon longirostris (Kaup, 1835)

Orden Artiodactyla Owen, 1848

Superfamilia Cervoidea Simpson, 1931

Familia Cervidae Gray, 1821

Cervidae indet.

Orden Carnivora Bowdich, 1821

Familia Hyaenidae Gray, 1869

Género Adcrocuta Kretzoi, 1938

Adcrocuta eximia (Roth y Wagner, 1855)

\section{TAFONOMÍA}

Se han obtenido 231 fósiles identificables, que representan el $15 \%$ de todo el material recuperado, ya que el resto corresponde a pequeños fragmentos o esquirlas imposibles de determinar, incluso a nivel anatómico.

Destaca, en este yacimiento, la selección que se produce para determinados elementos esqueléticos. Son mucho más abundantes los dientes (72\%) que los restos postcraneales. Estos últimos son, en su totalidad, elementos del autópodo (falanges, carpales/tarsales) o bien fragmentos epifisarios de huesos largos. Por lo tanto, todos ellos son de pequeño tamaño y forma redondeada, con un comportamiento hidrodinámico similar. 
Otra característica tafonómica del yacimiento de Tariego de Cerrato es el alto grado de abrasión que presentan la totalidad de los fósiles, de tal forma que en la mayor parte de los elementos postcraneales no es posible observar aristas que puedan proporcionar datos acerca de su procedencia anatómica y taxonómica.

Por todo lo comentado, se interpreta que la presencia de los fósiles en estos sedimentos es el resultado de una concentración debida a un transporte de origen hidrodinámico. Se trataría de una asociación de elementos resedimentados. Sin embargo, se descarta la posibilidad de reelaboración a partir de niveles previos datados con un Hipparion de edad más antigua que el presente en el yacimiento (Santisteban et al., 1997).

\section{CONCLUSIONES}

La asociación de macromamíferos presentes en el yacimiento de Tariego de Cerrato le asigna una edad correspondiente al Turoliense superior. El yacimiento se sitúa en unos materiales que corresponden a ambientes fluviales rodeados por áreas lagunares; concretamente, se localizaría dentro de canales efímeros. Los desbordamientos, relativamente frecuentes, debieron atravesar la llanura de inundación, arrastrando fragmentos óseos, tanto de los canales como de la propia llanura, pues aunque los fósiles muestran un elevado grado de abrasión producida por las partículas de arena arrastradas por la corriente, su estado de conservación es bueno.

\section{AGRADECIMIENTOS}

Este trabajo ha sido apoyado económicamente por la Consejería de Educación y Cultura de la Junta de Comunidades de Castilla y León, Proyecto: "Excavaciones Paleontólogicas en Tariego de Cerrato (Palencia)", $\mathrm{N}^{\circ}$ subv. 35.PA-06/95 y PA-05/97.

\section{BIBLIOGRAFÍA}

Christol, J. 1832. Comparaison de la population contemporaine des mammiferes de deux bassins du departement de 1'Herault. Annales de la Science et de l'Industrie du Midi de la France, 2, 180-181.

Falconer, H. and Cautley, P.T. 1845-1849. Fauna Antiquua Sivalensis, Being the Fossil Zoology of the Sewalik Hills in the North of India. Ed. London, Smith, Elder and Co., $307 \mathrm{pp}$.

Gervais, P. 1859. Zoologie et Paleontologie Francaises (Ed. A. Bertrand). Paris, 544 pp.

Kaup, J.J. 1832-39. Description des osements fossiles de Mammiferes inconus jusqu'a present qui se trouvent au Muséum grand ñ ducal de Darmstadt (Ed. J.P. Diehl). Darmstadt Cuad. IV, 65 - 89.

Kretzoi, M. 1938. Die Raubtiere von Gombaszog nebst einer ubersicht der Gesantfauna (ein Beitrag zur stratigrafische) des Alquart. Annales Musei National Hungaricum, 31, 88-157.

Mediavilla, R. y Dabrio, C.J. 1988. Controles sedimentarios neógenos en la Depresión del Duero (Sector Central). Revista de la Sociedad Geológica de España, 1, 195197.

Mediavilla, R.M., Alcalá, L., Santisteban, J.I., Alberdi, M.T., Luque, L., Mazo, A., Miguel, I., Morales, J. y Pérez, B. 1995. Estratigrafía y Paleontología del Mioceno superior del sur de la provincia de Palencia (Sector Central de la Cuenca del Duero). Comunicaciones XIII Congreso Español de Sedimentología. 153-154.

Pilgrim, G.E. 1926. The fossil Suidae of India. Paleontologica Indica, 8, 4, 1-65.

Pilgrim, G.E. 1937. Siwalik antelopes and oxen in the Americam Museum of Natural History. Bulletin American Museum of Natural History, 72, 729-874.

Roth, J. und Wagner, A. 1855. Die fossilen Knochen,berreste von Pikermi. Abhandlungen Bayerische Akademie Wissenschaften, 7, 371-464.

Santisteban, J.I., Alcalá, L.,Mediavilla, R.M., Alberdi, M.T., Luque, L., Mazo, A., Miguel, I., Morales, J. y Pérez, B. 1997. El yacimiento de Tariego de Cerrato: El inicio de la red fluvial actual en el Sector Central de la Cuenca del Duero. Cuadernos de Geología Ibérica, 22, 431-446. 